\title{
Reliability Analysis of Mechanical Sand Washing System
}

\author{
Yun-fei $\mathrm{Fu}^{1, \mathrm{a}}$, Jie Gong ${ }^{1, \mathrm{~b}}$, Zi-ming Yang ${ }^{1, \mathrm{c}^{\star}}, \mathrm{Pu}$-wang $\mathrm{Li}^{1, \mathrm{~d}}$, Si-dong $\mathrm{Li}^{1, \mathrm{e}}$, and \\ Ming-zhe $L v^{1, f}$
}
${ }^{1}$ Agricultural Product Processing Research Institute at Chinese Academy of Tropical Agricultural Sciences, Zhanjiang524001, China.
a18675991432@163.com, b18813611566@163.com, 'yangziming2004@163.com, dpuwangli@163.com, ${ }^{\mathrm{d}} 13702737491 @ 163 . c o m, f^{f} I v m z 12 @ 163 . c o m$

\begin{abstract}
Keywords: desalted sea sand; mechanical sand washing machine; sand washing process; system reliability; average life

Abstract. The purpose of this study is to calculate the reliability of the mechanical sand washing system. The basic principle and process flow of the sand washing system are introduced. According to the structural characteristic of the sand washing system, the simplified reliability model of the system is established. Based on the reliability model, the mathematical model of the system reliability is constructed. In the reliability analysis, the curve of system reliability is obtained. The results show that the maximum reliability of the system is approximate 0.9 , and the average life of the system is about 5.59 years.
\end{abstract}

\section{Introduction}

With the booming of economy in the world, the sand and building stones consumed in the construction field are increasing greatly [1]. At present, the river sand is the main source of the fine aggregate for building. But, due to the limitation of the resources and environments, the exploitation of the river sand are overwhelmingly restricted [2]. Therefore, the development and utilization of the sea sand are expected to solve the contradiction between the urban development and the restriction of the river sand. In China, there are exceedingly plentiful sea sand. According to preliminary evaluation, the storage capacity of the sea sand resources in the shallow sea is approximate $(67 \sim 100) \times 10^{8} \mathrm{~m}^{3}$. However, there are a couple of factors that limit the application of the sea sand. The main two factors are the shell and chloride salt. The chloride salt in sea sand will have great impact on the hydration process of the portland cement and will corrode the steel bar in the concrete [3]. Also, the shell will affect the strength and durability of the concrete.

To obtain the sand that meet the requirements of the construction engineering, various sorts of mechanical sand washing systems are adopted to wash the sea sand. Since the mechanical sand washing system is a intensely complex system with a plenty of elements, there are some breakdowns in the system, causing the economic loss and casualties. To ensure the reliable operation of the mechanical sand washing system, this study analyzes the reliability of a type of the mechanical sand washing system.

\section{Mechanical Sand Washing System}

In 2013, Q.Y. Tang, a scholar from Chinese Taiwan, presented a new mechanical sand washing system, the flow diagram of which is shown in Fig. 1 [4]. According to Fig. 1., it is manifest that the mechanical sand washing system mainly consists of the silo, vibrating screen, rotating wheel sand washing machine, screw wash-sand machine, recovery machine of fine sand, the dewatering screen. At present, the sand washing system shown in Fig. 1. has been widely used. The quality of the desalted sea sand and the production efficiency of the mechanical sand washing system shown in Fig. 1. meet the demands of the sea sand processing firms well. Thus, it is the most common sand washing system in China. This study takes the mechanical sand washing system presented by Q.Y. Tang as the research object. 


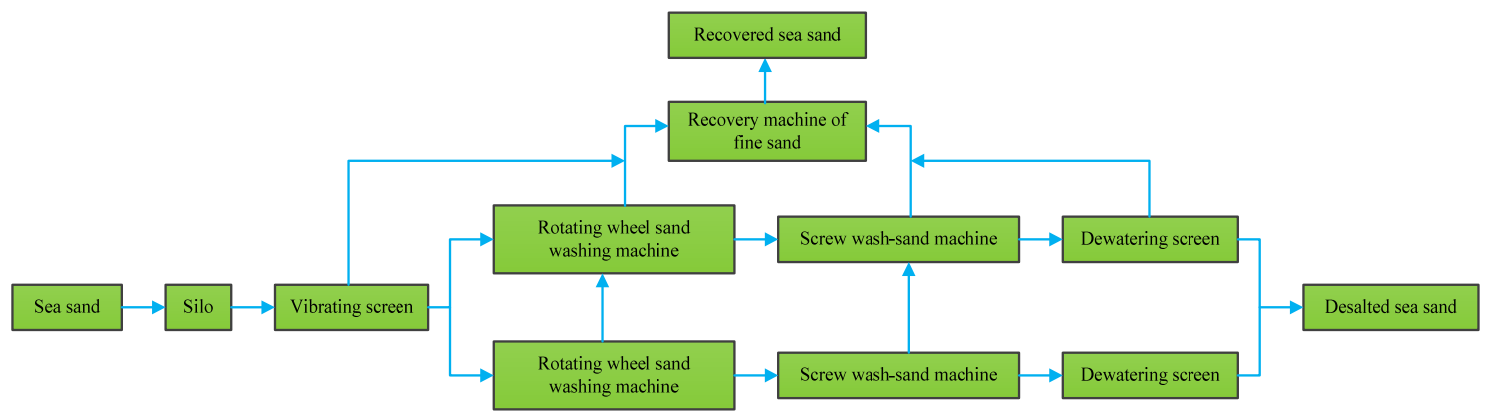

Fig. 1. Flow diagram of mechanical sand washing system

The process flow diagram of the mechanical sand washing system is shown in Fig. 2. Fig. 2 can illustrate the process of washing the sea sand clearly. First, the raw sea sand is transported to the vibrating screen. At the same time, the ozone water is poured into the screw wash-sand machine. At this stage, the shell and useless sundries can be removed. Next, the rotating wheel sand washing machine washes the sea sand, and uses the recovered ozone water to remove the clay from the sea sand. Next, by using the ozone water, the screw wash-sand machine removes the chloridion from the sea sand. Then, the dewatering screen is used to eliminate the water and the chloridion remaining on the surface from the sea sand. Meanwhile, the recovery machine of the fine sand absorbs the sea sand exhausted from the above processes. Finally, the desalted sea sand is obtained.

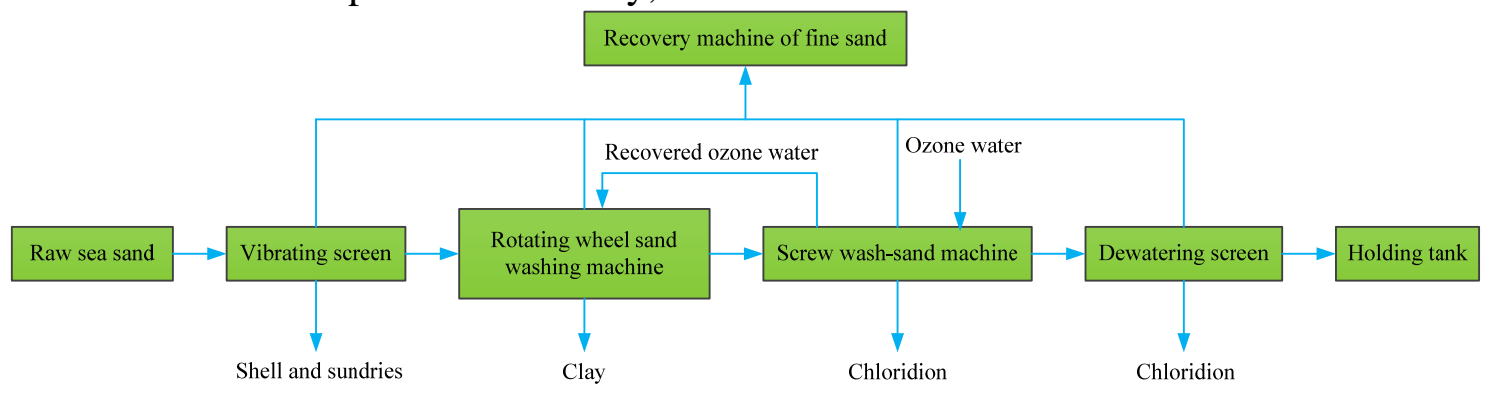

Fig. 2. Process flow diagram of mechanical sand washing system

The mechanical sand washing system has a couple of plus points listed as follow [4]:

(1) With the help of the silo and vibrating screen, the shell and sundries can be removed from the raw sea sand.

(2) The rotating wheel sand washing machine can utilize the recovered ozone water to remove the clay from the sea sand, which can save a large number of water.

(3) The screw wash-sand machine can use the ozone water to get rid of the chloridion.

(4) The dewatering screen can eliminate the chloridion remaining on the surface of the sea sand, and then the sand for building can be obtained.

(5) The recovery machine of the fine sand is capable of recovering the sea sand exhausted by sand washing machines, which can increase the yield and decline the loss rate of the sea sand.

\section{Establishment of System Reliability Model}

The reliability is an extremely crucial index that can be used to evaluate the product quality [5]. From the perspective of the pure economics, to reduce maintenance cost and improve the utilization ratio of products, the improvement of the reliability is overwhelmingly necessary $[5,6]$. But, when the reliability reaches the peak, the total consumption cost is not always the lowest. In addition, the manufacturing cost of products is a leading factor that should be taken into consideration. Therefore, the selection of the optimal reliability is intensely paramount. In other words, the study on the reliability is very necessary. As can be seen from Fig. 1, it is evident that the mechanical sand washing system is a typical compound system. In addition, it is notable that the reliability of the recovery machine of the fine sand has no significant effect on the reliability of the mechanical sand washing system, so establishment of the system reliability model will neglect the effect of the recovery machine of the fine sand. That is, the recovery machine of the fine sand is not a main element of the mechanical sand washing system. On the basis of the structural characteristic of the 
mechanical sand washing system, the reliability block diagram of the sand washing system is established, as shown in Fig. 3.

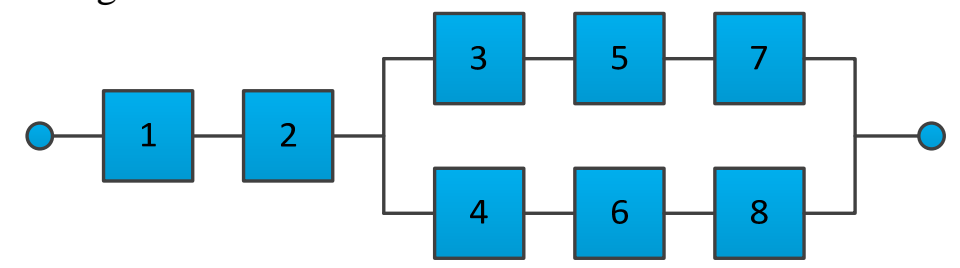

Fig. 3. Reliability block diagram of mechanical sand washing system

In Fig. 3, 1 denotes the silo; 2 denotes the vibrating screen; 3 and 4 denote rotating wheel sand washing machines; 5 and 6 denote screw wash-sand machines; 7 and 8 denote dewatering screens.

According to Fig. 3, the reliability of the mechanical sand washing system can be calculated as

$$
R_{s}=R_{1} \times R_{2} \times\left(1-\left(1-R_{3} \times R_{5} \times R_{7}\right) \times\left(1-R_{4} \times R_{6} \times R_{8}\right)\right)
$$

where $R_{1}$ is the reliability of the silo, $R_{2}$ is the reliability of the vibrating screen, $R_{3}$ and $R_{4}$ are the reliability of rotating wheel sand washing machines, $R_{5}$ and $R_{6}$ are the reliability of screw wash-sand machines, and $R_{7}$ and $R_{8}$ are the reliability of dewatering screens.

\section{Reliability Calculation of Mechanical Sand Washing System}

According to the engineering practice, the elements in the mechanical sand washing system follow the exponential distribution. The probability density function of the exponential distribution is $[7,8]$

$$
f(\mathrm{x})= \begin{cases}\lambda e^{-\lambda x} & x>0 \\ 0 & x \leq 0\end{cases}
$$

where $\lambda$ is the failure rate of the element.

The reliability of the mechanical sand washing system can also be expressed by

$$
R_{s}=e^{-\left(\lambda_{1}+\lambda_{2}\right) t} \times\left(1-\left(1-e^{-\left(\lambda_{3}+\lambda_{5}+\lambda_{7}\right) t}\right) \times\left(1-e^{-\left(\lambda_{4}+\lambda_{6}+\lambda_{8}\right) t}\right)\right)
$$

The failure rates of different elements are listed in Tab. 1 ,

\begin{tabular}{ccccccccc}
\hline Tab. 1. The failure rates of elements \\
\hline Element & $\lambda_{1}$ & $\lambda_{2}$ & $\lambda_{3}$ & $\lambda_{4}$ & $\lambda_{5}$ & $\lambda_{6}$ & $\lambda_{7}$ & $\lambda_{8}$ \\
\hline${\text { Failure rate } / \mathrm{h}^{-1}}^{0.000015}$ & 0.000005 & 0.00025 & 0.00025 & 0.0005 & 0.0005 & 0.00045 & 0.00045 \\
\hline
\end{tabular}

The curve of the reliability of the mechanical sand washing system is shown in Fig. 4.

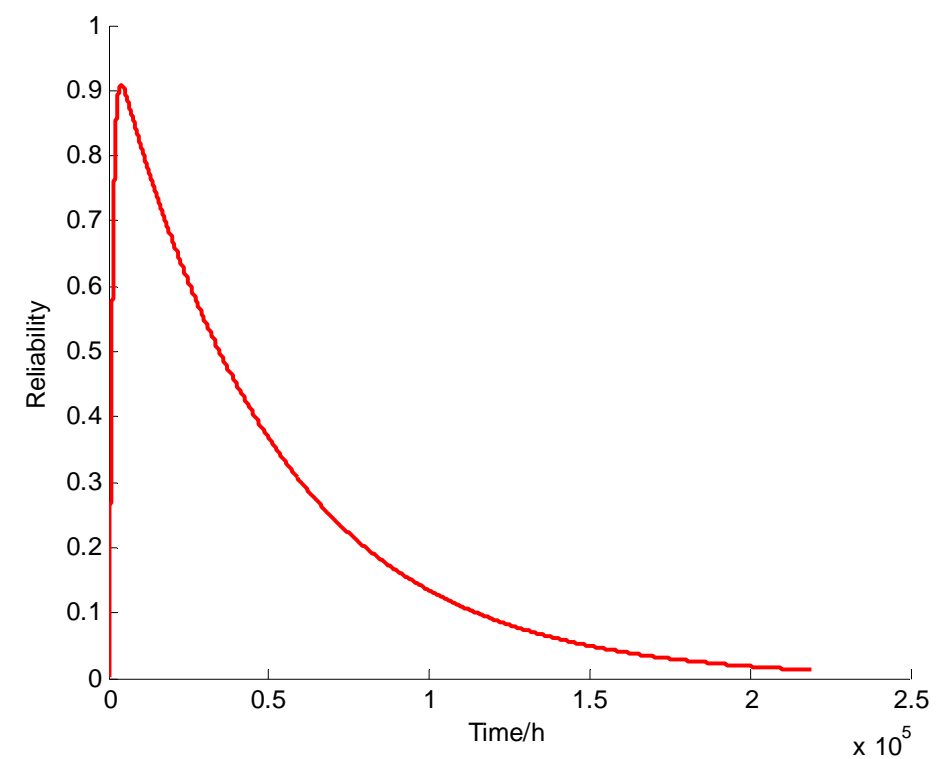

Fig. 4. Curve of system reliability 
It can be seen from Fig. 4 that from 0 to $0.1 \times 10^{5}$ hours, the curve experiences a sharp growth, peaking at 0.9 , and the line graph suffers from a rapid downward trend. The average life of the mechanical sand washing system is given by

$$
\theta_{s}=\int_{0}^{+\infty} R_{s}(t) d t
$$

By calculating the Eq. (4), it is known that the average life of the mechanical sand washing system is $4.9 \times 10^{4}$ hours, 5.59 years.

\section{Conclusions}

(1) The sand washing system presented in this paper is a typical compound system.

(2) The maximum reliability of the mechanical sand washing system is approximate 0.9.

(3) The mean life of this kind of sand washing system is about $4.9 \times 10^{4}$ hours, 5.59 years.

(4) The study provides some theoretical basis for the reliability optimization design of the mechanical sand washing system.

\section{Acknowledgements}

The authors gratefully acknowledged the Financial Support by the Foundation of Science and Technology Competitive Allocation of Zhanjiang (No. 2014A02010), the Funds for Innovation Introduced and Integration Project of Hainan Province (No. KJHZ2014-10).

\section{References}

[1] H.G. Yin, Y. Li, H. Lv and Q. Gao: Mining Science and Technology (China) Vol. 21 (2011), p. 123

[2] O.E. Gjørv: Arabian Journal for Science and Engineering Vol. 36 (2011), p. 151

[3] R. Vedalakshmi, R.R. Devi, B. Emmanuel and N. Palaniswamy: Materials and Structures Vol. 41 (2008), p. 1315

[4] Q.Y. Tang, C.N. Patent 203484221 U. (2014)

[5] S.K. Choi, R.V. Grandhi and R.A. Canfield: Reliability-Based Structural Design (Springer, London 2010).

[6] X.L. Yang: Reliability Design (Higher Education Press, Beijing 2013).

[7] Q.G. Hu and Y.P. Liu: Design and Application of Mechanical Reliability (Publishing House of Electronics Industry, Beijing 2014).

[8] J.J. Xiong and R.A. Shenoi: Fatigue and Fracture Reliability Engineering (Springer, New York 2011). 